\title{
Evaluation of Ergonomic Risk of Manual Handling of Patients With MAPO Index and Its Relationship with Incidence of Low Back Pain among Nurses
}

\author{
Mahnaz Saremi ${ }^{1, a^{*}}$, Fatemeh Khayati ${ }^{2, b}$ \\ ${ }^{1,2}$ Faculty of Health, Safety and Environment, Shahid Beheshti University of Medical Sciences, \\ Tehran, Iran \\ a*m.saremi@sbmu.ac.ir, ${ }^{\text {b} F k h a y a t .90 @ g m a i l . c o m ~}$
}

Keywords: ergonomics risk, MAPO index, Low Back Pain, nurses, manual patients handling.

\begin{abstract}
Low back pain (LBP) is the most common and most costly musculoskeletal disorder among nursing profession. The ergonomic risk factors are common causes of lumbar injury. This study aims to assess the risk of LBP among nurses in different wards of an Iranian subspecialty hospital by means of MAPO method and to investigate if there is any relationship between calculated index and the self-reported LBP. The study was conducted in 16 sections of an Iranian subspecialty hospital including 174 wards. Exposed nursing staff $(\mathrm{N}=54)$ were asked to report LBP experiences in the past year. Our results indicated that approximately $90 \%$ of examined wards were in the red band while only $9 \%$ were in the yellow and $1 \%$ in the green bands. Among all nurses who worked in the high risk wards, $25 \%$ reported suffering LBP during the past 12 months. However, $16 \%$ of those who were in the medium risk wards also experienced LBP in the past year. Chi-square test showed a significant relationship between LBP incidence and the level of MAPO index $(p=0.004)$. This study suggests that the frequency of LBP among nurses is higher than the general population. The fact that most of the studied wards felt in the red band indicates the elevated risk for low back in the majority of the hospital wards. It is therefore necessary to implement an ergonomic program in order to modify the observed deleterious condition as soon as possible.
\end{abstract}

\section{Introduction}

Musculoskeletal Disorders (MSDs) represent a common occupational problem in the nursing profession and a regular topic for safety research [1]. Musculoskeletal problems are common among the general and working population [2] and can result in serious social and economic impacts on both individuals and communities [2-4]. The United Kingdom's Health and Safety Executive estimated that, in 2009 - 2010, musculoskeletal disorders were the most commonly reported occupational illness and the main cause for approximately $37 \%$ of working days lost [2]. Low back pain (LBP) is the most common and most costly musculoskeletal disorder; so that, it is recognized as a major public health problem throughout the world $[3,5]$. Previous researches have relied on two main criteria to assess musculoskeletal injury risk exposure: those based on epidemiological investigations and those based on analysis of the potential biomechanical overload of the lumbar disks. Concerning manual patient handling, epidemiological studies point unequivocally to the existence of a relationship between the type and number of maneuvers involved in manual movement of patients and the occurrence of certain acute and chronic disorders of the lumbar spine. However, this task often produces a disk load exceeding the values defined as tolerable [6]. In general, musculoskeletal disorders (MSDs) are known to be related with physical and psychosocial job demands, such as heavy load lifting, repetitive tasks, awkward working postures and stress $[7,8]$. Menoni and colleagues have developed a risk assessment method called Movement and Assistance of Hospital Patients (MAPO), which is applicable in hospital wards in order to assess the risk exposure level of manual patient handling. This quantitative method is not only a practical tool for analysis, but also for intervention and prevention [6,9].

This study is the first attempt to evaluate the risk of manual patient handling in Iranian hospital wards. We hypothesized that the MAPO index is related to the incidence of LBP among nurses. So, the higher prevalence of LBP is expected in the wards in which the index value is much higher. 


\section{Methods}

This study was carried out in a subspecialty hospital in Tehran, Iran. Sixteen sections of the hospital (ICU, CCU, NICU, BICU, Internal medicine, Surgery, cardiology, Transplantation, Pediatric and Emergency rest cardiology, Obstetricians) including 174 wards were included in the study. The total number of nurses involved in manual patients handling in the studied sections was 54 (aged from 25 to 43 years; mean $33 \pm 5.05$ years). All of them were invited to participate to the study.

MAPO method was applied in order to assess the risk of manual patients handling. Calculating the MAPO index needs a data recording sheet (Menoni et al. 2004) consisting of two parts [6]. The first part collects all information concerning organization and training aspects through an interview with the head nurse. The second part is specifically designed for the analysis of environmental and equipment aspects and for assessment of specific subsidiary maneuvers through an on-site inspection [9].

This protocol $[6,9]$ allows identification of factors necessary for calculation of the MAPO index:

Disabled patient/operator ratios (NC/OP and PC/OP): It is necessary to know the number of workers employed in the unit, and assigned to manual patient handling, to give the ratio of the average number of disabled patients present in the unit to the sum of presences across the three shifts (the total number of operators; OP). Once the average number of disabled patients is known, they are further classified into totally non-cooperative (NC) and partially cooperative (PC) patients on the basis of their residual motor capacity and current illness.

Lifting factor (LF): Assessment of patient lifting devices combines two aspects: sufficiency (i.e. at least one lifting device for every eight $\mathrm{NC}$ patients); and adequacy of lifting devices. A lifting device is considered as inadequate if it cannot be used for the type of patient normally present in the ward; is in a poor state of repair (often broken); or cannot be used due to the environmental features of the wards and/or bathrooms. The value assigned to the LF varies from 0.5 to 4 .

Minor aid factor (AF): A minor aid is considered to be a piece of equipment reducing lifting frequency and/or biomechanical overload produced by certain operations to partially move the patient (e.g. sliding sheet, transfer disc, roller and ergonomic belt). Such minor aids are considered to be present when the unit is equipped with one sliding sheet plus at least two of the other aids mentioned. A reducing value (0.5) is then assigned to the factor, considering that the presence of these aids reduces the number of such operations. When minor aids are not present or are insufficient, the value assigned is 1 .

Wheelchair factor (WF): Assessment of wheelchairs and/or commodes considers two aspects in an integrated manner: sufficient number as compared to the number of disabled patients; and presence of ergonomic requirements. A sufficient number means the presence of a number of wheelchairs equal to at least half of the number of disabled patients in the unit.

For each type of wheelchair/commode, identified during on-site inspection, features are assessed by assigning each one a value of 1 if absent (with a maximum of 4). The sum of the inadequacy score for each type of wheelchair, multiplied by the number of wheelchairs (with the same features), gives the total score for each type of wheelchair. The sum of the different column scores, divided by the total number of wheelchairs, gives the mean wheelchair score. This score summarizes the ergonomic appropriateness of all wheelchairs/commodes present in the unit.

It is thus possible to define the WF value by combining the two assessed aspects (sufficient number and ergonomic requirements). The value of this factor varies from 0.75 to 2 .

Environment factor (EF): This factor covers analysis of bathrooms, toilets and wards. For each section, the number of inadequacy features is identified. The highest scores (1 or 2$)$ are assigned to environmental aspects which, if inadequate, enforce the operators to perform a higher number of manual patient transfer. The lowest score $(0.5)$ is assigned to presence of furniture (e.g. 
armchairs) not allowing the PC patient to use any residual motor capacity that they have, so that the operator has to lift the patient. For each section (bathrooms, toilets, wards) the procedure is the same as for wheelchairs, calculating the mean score of section inadequacy. The sum of the mean scores of the three sections makes up the mean environment score, which is divided into three categories of equidistant range, expressing low, medium and high inadequacy. The value of the EF varies from 0.75 to 1.5 .

Training factor (TF): The last factor determines the specific training of operators. A reducing value of 0.75 was assigned to the cases of adequate training (theoretical and practical exercises on techniques for lifting PC patients with the least overload). When training simply provided information (verbally or via leaflets), a TF of 1 was assigned. When no training was given, a TF of 2 was assigned.

Then, using the following formula, the ergonomic risk of manual handling of patients in each ward (MAPO index) can be calculated.

\section{MAPO $=[(\mathbf{N C} / \mathbf{O P} \times \mathbf{L F})+(\mathbf{P C} / \mathrm{OP} \times \mathbf{A F})] \times \mathbf{W F} \times \mathbf{E F} \times \mathbf{T F}$}

According to Menoni et al. (2004), final MAPO index would be classified into three categories: $0-1.5$ (green band); $1.51-5.00$ (yellow band); 5.01- and greater (red band). The risk is negligible if the band is green. In this case, the prevalence of work-related LBP could be estimated to be identical to those of the general population (3.5\%). The yellow 'alert' band shows that low back pain may have an incidence 2.4 times higher than the green band. At this level, it is necessary to make a medium- and long-term intervention plan for health surveillance, aid equipment and training. The red band, with exposure index above 5, corresponds to a higher risk, where low back pain may have an incidence up to 5.6 times higher the expected incidence. In this case, an immediate intervention plan must be made for health surveillance, aid equipment, training and environmental improvement [6,9].

Data concerning the incidence of LBP was also collected by means of a questionnaire.

\section{Results}

Results are summarized in Table 1. Among all studied wards, only one (NICU) felt in the green band. The others were either in the yellow (MAPO indexes vary from 1.80 to 4.80 ) or in the red band (MAPO indexes vary from 5.39 to 13.12).

Table 1. MAPO Index values and classification bands for different sections.

\begin{tabular}{clcc}
\hline No & section & $\begin{array}{c}\text { MAPO Index } \\
\text { value }\end{array}$ & $\begin{array}{c}\text { MAPO } \\
\text { classification }\end{array}$ \\
\hline 1 & NICU & 1.31 & Green band \\
2 & ICU & 4.80 & Yellow band \\
3 & CCU & 4.20 & Yellow band \\
4 & BICU & 3.00 & Yellow band \\
5 & ICU-OH & 2.25 & Yellow band \\
6 & Cardiology (w) & 4.10 & Yellow band \\
7 & Obstetricians & 1.80 & Yellow band \\
8 & Cardiology (m) & 5.39 & Red band \\
9 & Surgery (w) & 8.31 & Red band \\
10 & Surgery (m1) & 6.02 & Red band \\
11 & Surgery (m2) & 8.49 & Red band \\
12 & Internal medicine (w) & 6.37 & Red band \\
13 & Internal medicine (m) & 9.71 & Red band \\
14 & Transplantation & 8.90 & Red band \\
15 & Pediatrics & 13.12 & Red band \\
16 & Emergency rest & 6.56 & Red band \\
\hline
\end{tabular}

NICU: New Born Intensive Care Unit; ICU: Intensive Care Unit; CCU: Cardiac Care Unit;

BICU: Burn Intensive Care Unit; ICU-OH: Intensive Care Unit - Open-heart surgery; m: special section for men; w: special section for women. 
Figure 1. shows that approximately $90 \%$ of all of the examined wards were in the red band while only $9 \%$ were in the yellow and $1 \%$ in the green bands. Among all nurses who worked in the high risk wards, $25 \%$ reported suffering LBP during the past 12 months. However, $16 \%$ of those who were in the medium risk wards also experienced LBP in the past year.

Chi-square test showed a significant relationship between LBP incidence and the level of MAPO index $(p=0.004)$.

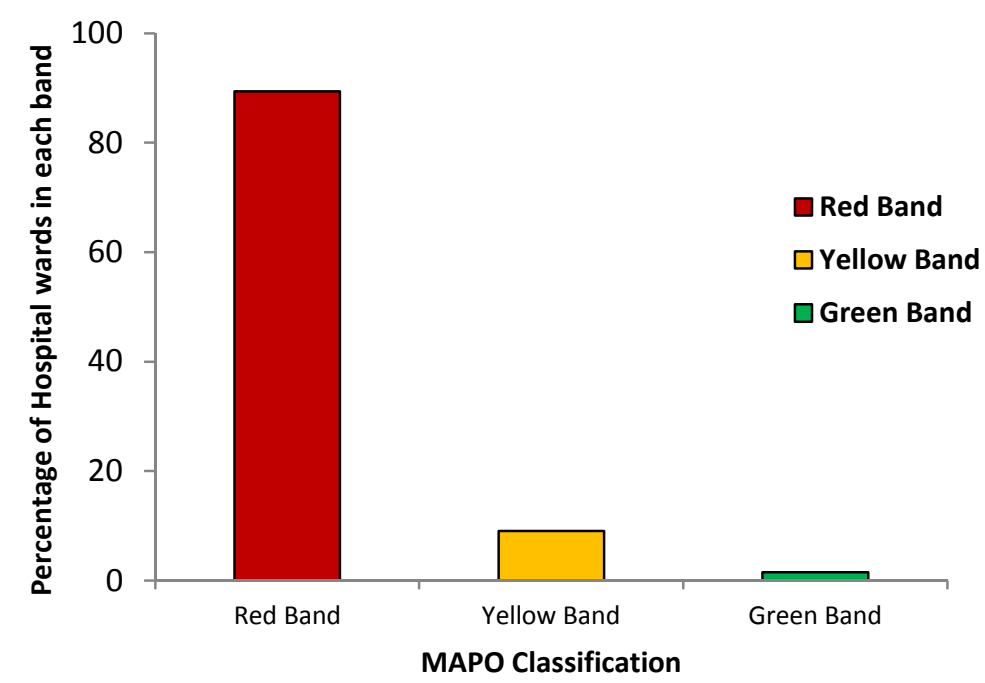

Fig. 1. Distribution of hospital wards according to the three level of MAPO index.

Using the K - S test, normal distribution of data was examined ( $\mathrm{P}>0.05$; see Table 2$)$. Then, the relationship between ward variables with MAPO index was examined by multiple regression test. Based on these results, the wheelchair factor $(\mathrm{WF})(\mathrm{r}=0.338)$ and environment factor $(\mathrm{EF})(\mathrm{r}=\mathrm{o.137})$ could be the most effective variables to decrease the risk level. NC/OP factor $(\mathrm{r}=0.132), \mathrm{PC} / \mathrm{OP}$ factor $(\mathrm{r}=0.129)$, TF $(\mathrm{r}=0.123), \mathrm{AF}(\mathrm{r}=0.108)$ and $\mathrm{LF}(\mathrm{r}=0.101)$ were found to be at next priority levels; respectively.

Table 2. Normal distribution of studied variables.

\begin{tabular}{lc}
\hline Variables & P-values $^{*}$ \\
\hline NC/OP & 0.808 \\
PC/OP & 0.590 \\
AF & 0.102 \\
LF & 0.100 \\
TF & 0.117 \\
WF & 0.228 \\
EF & 0.746 \\
MAPO Index & 0.996 \\
\hline One -sample Kolmogorov- Smimov Test
\end{tabular}

\section{Discussion}

The aim of this study was to investigate if the ergonomic risk of manual patients handling in hospital wards is associated with the experience of low back pain in exposed staff. The prevalence of low back pain in general populations of different countries has been previously reported. Accordingly, 3.5\% of general Italian population (Menoni et al. 2004), $8.4 \%$ of general Indian population (Mahajan et al. 2003) and 9.5\% of Kuwaitis (Al-Awadhi et al. 2004) suffer from LBP $[6,10,11]$. In general Iranian population, the prevalence of low back pain has been reported about $14.8 \%$ by Davatchi et al. (2008), and more recently was confirmed by another research [12,13]. Our results revealed that the frequency of LBP among nurses is higher than the general population. This 
finding is in accordance with the results of previous researches on this topic, since a prevalence rate of 51\% has already been estimated for LBP in Iranian nursing staff $[14,15]$.

Only $1 \%$ of wards were identified to have no risk for LBP. The most studied wards felt in the red band, indicating the elevated risk for low back in the majority of hospital wards. It is therefore necessary to implement an ergonomic program in order to modify the observed deleterious condition as soon as possible. The main effective factors for reducing the index value could be environment factor and wheelchair factor. Wards, bathrooms and toilets are each one a component of Environment factor which should be ergonomically modified. Moreover, preparing assistive patient handling devices could certainly be effective. Unfortunately, wheelchairs were not only insufficient but also always unavailable or damaged. So, a proper maintenance should strictly be planned. Moreover, the number of employees seems to be insufficient with regards to disable patients. Increasing the number of nurses would results in the lower final index which in turn could reduce LBP. These findings enabled us to evaluate the risk of LBP in the various hospital wards. The second step is to prepare an effective preventive program in order to reduce the risk of low back disorders in care-assistants.

\section{References}

[1] M.Z.O. Coluci, N.M.C. Alexandre, J. Rosecrance, Reliability and validity of an ergonomicsrelated Job Factors Questionnaire, Int. J. Ind. Ergon. 39 (2009) 995-1001.

[2] B. Widanarko, S. Legg, M. Stevenson, J. Devereux, A. Eng, A. Mannetje, S. Cheng, J. Douwes, L. Ellison-Loschmann, D. McLean and N. Pearce, Prevalence of musculoskeletal symptoms in relation to gender, age, and occupational/industrial group, Int. J. Ind. Ergon. 41 (2011) 561-572.

[3] V. Gouttebarge, H. Wind, P. P Kuijer, J.K. Sluiter, M.H. Frings-Dresen, Reliability and agreement of 5 ergo-kit functional capacity evaluation lifting tests in subjects with low back pain, Arch. Phys. Med. Rehabil. 87 (2006) 1365-1370.

[4] N.A. Roussel, J. Nijs, S. Truijen, L. Smeuninx, and G. Stassijns, Low back pain: clinometric properties of the trendelenburg test, active straight leg raise test, and breathing pattern during active straight leg raising, J. Manipulative Physiol. Ther. 30 (2004) 270-278.

[5] H. Carlsson, E. Rasmussen-Barr, Clinical screening tests for assessing movement control in non-specific low-back pain. A systematic review of intra- and inter-observer reliability studies, J. Manual therapy. 18 (2013) 103-110.

[6] N. Stanton, A. Hedge, K. Brookhuis, E. Salas, H. Hendrick, Handbook of human factors and ergonomics methods. Second ed., CRC, New York, 2005.

[7] A. Choobineh, M. Motamedzade, M. Kazemi, A. Moghimbeigi, A. Heidari Pahlavian, The impact of ergonomics intervention on psychosocial factors and musculoskeletal symptoms among office workers, Int. J. Ind. Ergon. 41 (2011) 671-676.

[8] D.R. Smith, M. Mihashi, Y. Adachi, H. Koga, T. Ishitake, Detailed analysis of musculoskeletal disorder risk factors among Japanese nurses, J. Safety Res. 37 (2006) 195 - 200.

[9] N. Battevi, O. Menoni, M. Grazia Ricci, S. Cairoli, MAPO index for risk assessment of patient manual handling in hospital wards: a validation study, Ergonomics 49 (2006) 671-687.

[10] A. Mahajan, D.S. Jasrotia, A.S. Manhas, S.S. Jmwal, Prevalence of major rheumatic disorders in Jammu. J. K. Science. 5 (2003) 63-66.

[11] A.M. Al-Awadhi, S.O. Olusi, K. Al-Saeid, M. Moussa, D. Shehab, N. Al-Zaid, A. Al-Herz, K. Al-Jarallah, Musculoskeletal pain, disability and health-seeking behavior in adult Kuwaitis using a validated Arabic version of WHO-ILAR COPCORD Core Questionnaire. Clin. Exp. Rheumatol. 22 (2004) 177-183. 
[12] F. Davatchi, A.R. Jamshidi, A.T. Banihashemi, WHO-ILAR COPCORD study (stage 1, urbanstudy) in Iran. J. Rheum. 35 (2008) 1384-1390.

[13] S.J. Mousavi , M.E. Akbari, H. Mehdian, B. Mobini, A. Montazeri, B. Akbarnia, M. Parnianpour. Low back pain in Iran: a growing need to adapt and implement evidence-based practice in developing countries. Spine 36 (2011) 638-46.

[14] M.A. Mohseni Bandpey, M. Fakhri, M. A. Shirvani, M. Bagheri Nezami, A. Khalilian, Epidemiological aspects of low back pain in nurses, J. Babol. Univ. 7 (2005) 35-40 [in Persian].

[15] M. Hosseini, S. Varmazyar, A. Safari, Assess the physical status of emergency personnel of Qazvin teaching hospitals with rapid assessment of the entire body method and its relationship to musculoskeletal disorders, J Qom Univ. 4 (2010) 32-39 [in Persian]. 